International Mathematical Forum, Vol. 9, 2014, no. 7, 305 - 316

HIKARI Ltd, www.m-hikari.com

http://dx.doi.org/10.12988/imf.2014.38159

\title{
Cohomology Associated to a Poisson Structure on Weil Bundles
}

\author{
Vann Borhen Nkou ${ }^{1,2}$ and Basile Guy Richard Bossoto ${ }^{1}$ \\ ${ }^{1}$ Marien NGOUABI University, Faculty of Science \\ Department of Mathematics \\ BP: 69 - Brazzaville, Congo \\ ${ }^{2}$ Abomey Calavi University, IMSP \\ BP: 13, Porto-novo, Benin
}

Copyright (c) 2014 Vann Borhen Nkou and Basile Guy Richard Bossoto. This is an open access article distributed under the Creative Commons Attribution License, which permits unrestricted use, distribution, and reproduction in any medium, provided the original work is properly cited.

\begin{abstract}
Let $M$ be a paracompact smooth manifold of dimension $n, A$ a Weil algebra and $M^{A}$ the Weil bundle associated. We define and describe the notion of $\widetilde{d}$-Poisson cohomology and of $\widetilde{d_{A}}$-Poisson cohomology on $M^{A}$.

Mathematics Subject Classification: 58A20, 58A32, 17D63, 53D17, 53D05
\end{abstract}

Keywords: Weil bundle, Weil algebra, Poisson manifold, cohomology

\section{Introduction}

A local algebra in the sens of André Weil or simply a Weil algebra is a real, unitary, commutative algebra of finite dimension with a unique maximal ideal of codimension 1 on $\mathbb{R}[10]$.

Let $A$ be a Weil algebra and $\mathfrak{m}$ be its maximal ideal. We have

$$
A=\mathbb{R} \oplus \mathfrak{m} .
$$


The first projection

$$
A=\mathbb{R} \oplus \mathfrak{m} \longrightarrow \mathbb{R}
$$

is a homomorphism of algebra which is surjective, called augmentation and the unique none-zero integer $k \in \mathbb{N}$ such that $\mathfrak{m}^{k} \neq(0)$ and $\mathfrak{m}^{k+1}=(0)$ is the height of $A$.

If $M$ is a smooth manifold, $C^{\infty}(M)$ the algebra of differentiable functions on $M$ and $A$ a Weil algebra of maximal ideal $\mathfrak{m}$, an infinitely near point to $x \in M$ of kind $A$ is a homomorphism of algebras

$$
\xi: C^{\infty}(M) \longrightarrow A
$$

such that $[\xi(f)-f(x)] \in \mathfrak{m}$ for any $f \in C^{\infty}(M)$.

We denote $M_{x}^{A}$ the set of all infinitely near points to $x \in M$ of kind $A$ and

$$
M^{A}=\bigcup_{x \in M} M_{x}^{A}
$$

The set $M^{A}$ is a smooth manifold of dimension $\operatorname{dim} M \times \operatorname{dim} A$ called manifold of infinitely near points of kind $A[6]$.

When both $M$ and $N$ are smooth manifolds and when

$$
h: M \longrightarrow N
$$

is a differentiable application, then the application

$$
h^{A}: M^{A} \longrightarrow N^{A}, \xi \longmapsto h^{A}(\xi),
$$

such that, for any $g \in C^{\infty}(N)$,

$$
\left[h^{A}(\xi)\right](g)=\xi(g \circ h)
$$

is also differentiable. When $h$ is a diffeomorphism, it is the same for $h^{A}$.

Moreover, if $\varphi: A \longrightarrow B$ is a homomorphism of Weil algebras, for any smooth manifold $M$, the application

$$
\varphi_{M}: M^{A} \longrightarrow M^{B}, \xi \longmapsto \varphi \circ \xi
$$

is differentiable. In particular, the augmentation

$$
A \longrightarrow \mathbb{R}
$$


defines for any smooth manifold $M$, the projection

$$
\pi_{M}: M^{A} \longrightarrow M
$$

which assigns every infinitely near point to $x \in M$ to its origin $x$. Thus $\left(M^{A}, \pi_{M}, M\right)$ defines the bundle of infinitely near points or simply weil bundle $[7],[4],[10]$.

If $(U, \varphi)$ is a local chart of $M$ with coordinate functions $\left(x_{1}, x_{2}, \ldots, x_{n}\right)$, the application

$$
U^{A} \longrightarrow A^{n}, \xi \longmapsto\left(\xi\left(x_{1}\right), \xi\left(x_{2}\right), \ldots, \xi\left(x_{n}\right)\right)
$$

is a bijection from $U^{A}$ into an open of $A^{n}$. The manifold $M^{A}$ is a smooth manifold modeled over $A^{n}$, that is to say an $A$-manifold of dimension $n$ [1], [9].

The set, $C^{\infty}\left(M^{A}, A\right)$ of differentiable functions on $M^{A}$ with values in $A$ is a commutative, unitary algebra over $A$. When one identitifies $\mathbb{R}^{A}$ with $A$, for $f \in C^{\infty}(M)$, the application

$$
f^{A}: M^{A} \longrightarrow A, \xi \longmapsto \xi(f)
$$

is differentiable. Moreover the application

$$
C^{\infty}(M) \longrightarrow C^{\infty}\left(M^{A}, A\right), f \longmapsto f^{A},
$$

is an injective homomorphism of algebras and we have:

$$
(f+g)^{A}=f^{A}+g^{A} ;(\lambda \cdot f)^{A}=\lambda \cdot f^{A} ;(f \cdot g)^{A}=f^{A} \cdot g^{A}
$$

for $\lambda \in \mathbb{R}, f$ and $g$ belonging to $C^{\infty}(M)$.

We denote $\mathfrak{X}\left(M^{A}\right)$, the set of all vector fields on $M^{A}$. According to [1], We have the following equivalent assertions:

1. $X: C^{\infty}\left(M^{A}\right) \longrightarrow C^{\infty}\left(M^{A}\right)$ is a vector field on $M^{A}$;

2. $X: C^{\infty}(M) \longrightarrow C^{\infty}\left(M^{A}, A\right)$ is a linear application which verifies

$$
X(f g)=X(f) \cdot g^{A}+f^{A} \cdot X(g)
$$

for any $f, g \in C^{\infty}(M)$ i.e is a derivation of $C^{\infty}(M)$ into $C^{\infty}\left(M^{A}, A\right)$ with respect to the module structure

$$
C^{\infty}\left(M^{A}, A\right) \times C^{\infty}(M) \longrightarrow C^{\infty}\left(M^{A}, A\right),(\varphi, f) \longmapsto \varphi \cdot f^{A} .
$$

Thus, the set $\mathfrak{X}\left(M^{A}\right)$ of all vector fields on $M^{A}$ is a $C^{\infty}\left(M^{A}, A\right)$-module. 
When

$$
\theta: C^{\infty}(M) \longrightarrow C^{\infty}(M)
$$

is a vector field on $M$, the application

$$
\theta^{A}: C^{\infty}(M) \longrightarrow C^{\infty}\left(M^{A}, A\right), f \longmapsto[\theta(f)]^{A},
$$

is a vector field on $M^{A}$. The vector field $\theta^{A}$ is the prolongation to $M^{A}$ of the vector field $\theta$.

Theorem 1 If $X$ is a vector field on $M^{A}$ considered as a derivation of $C^{\infty}(M)$ into $C^{\infty}\left(M^{A}, A\right)$, then there exists, an unique derivation

$$
\widetilde{X}: C^{\infty}\left(M^{A}, A\right) \longrightarrow C^{\infty}\left(M^{A}, A\right)
$$

such that such that

1. $\widetilde{X}$ is A-linear;

2. $\widetilde{X}\left[C^{\infty}\left(M^{A}\right)\right] \subset C^{\infty}\left(M^{A}\right)$;

3. $\tilde{X}\left(f^{A}\right)=X(f)$ for any $f \in C^{\infty}(M)$.

Thus, the application

$$
[,]: \mathfrak{X}\left(M^{A}\right) \times \mathfrak{X}\left(M^{A}\right) \longrightarrow \mathfrak{X}\left(M^{A}\right),(X, Y) \longmapsto \widetilde{X} \circ Y-\widetilde{Y} \circ X,
$$

is $A$-bilinear and defines a structure of Lie algebra over $A$ on $\mathfrak{X}\left(M^{A}\right)[1]$.

The goal of this paper is to define and describe the notion of $\widetilde{d}$-Poisson cohomology and of $\widetilde{d_{A}}$-Poisson cohomology.

\section{Poisson structure on Weil bundles}

In this section, $M$ is a Poisson manifold i.e there exists a bracket $\{$,$\} on C^{\infty}(M)$ such that the pair $\left(C^{\infty}(M),\{\},\right)$ is a real Lie algebra and for any $f \in C^{\infty}(M)$, the application

$$
a d(f): C^{\infty}(M) \longrightarrow C^{\infty}(M), g \longmapsto\{f, g\}
$$

is a derivation of commutative algebra i.e

$$
\{f, g \cdot h\}=\{f, g\} \cdot h+g \cdot\{f, h\}
$$


for $f, g, h \in C^{\infty}(M)[5],[8]$.

We denote

$$
C^{\infty}(M) \longrightarrow \operatorname{Der}_{\mathbb{R}}\left[C^{\infty}(M)\right], f \longmapsto a d(f)
$$

the adjoint representation and $d$ the operator of cohomology associated to this representation. For any $p \in \mathbb{N}$,

$$
\Lambda_{\text {Pois }}^{p}(M)=C^{p}\left[C^{\infty}(M), C^{\infty}(M)\right]
$$

denotes the $C^{\infty}(M)$-module of skew-symmetric multilinear forms of degree $p$ from $C^{\infty}(M)$ into $C^{\infty}(M)$. We have

$$
\Lambda_{\text {Pois }}^{0}(M)=C^{\infty}(M)
$$

The $A$-algebra $C^{\infty}\left(M^{A}, A\right)$ is a Poisson algebra over $A$ if there exists a bracket $\{$,$\} on C^{\infty}\left(M^{A}, A\right)$ such that the pair $\left(C^{\infty}\left(M^{A}, A\right),\{\},\right)$ is a Lie algebra over $A$ satisfying

$$
\left\{\varphi_{1} \cdot \varphi_{2}, \varphi_{3}\right\}=\left\{\varphi_{1}, \varphi_{3}\right\} \cdot \varphi_{2}+\varphi_{1} \cdot\left\{\varphi_{2}, \varphi_{3}\right\}
$$

for any $\varphi_{1}, \varphi_{2}, \varphi_{3} \in C^{\infty}\left(M^{A}, A\right)[3],[2]$.

When $M$ is a Poisson manifold with bracket $\{$,$\} , for any f, g \in C^{\infty}(M)$,

$$
a d(f g)=a d(f) \cdot g+f \cdot a d(g) .
$$

For any $f \in C^{\infty}(M)$, let

$$
[a d(f)]^{A}: C^{\infty}(M) \longrightarrow C^{\infty}\left(M^{A}, A\right), g \longmapsto\{f, g\}^{A},
$$

be the prolongation of the vector field $a d(f)$ and let

$$
\widetilde{[a d(f)}]^{A}: C^{\infty}\left(M^{A}, A\right) \longrightarrow C^{\infty}\left(M^{A}, A\right)
$$

be the unique $A$-linear derivation such that

$$
\widetilde{[a d(f)}]^{A}\left(g^{A}\right)=[\operatorname{ad}(f)]^{A}(g)=\{f, g\}^{A}
$$

for any $g \in C^{\infty}(M)$.

Theorem 2 [3] For $\varphi \in C^{\infty}\left(M^{A}, A\right)$, the application

$$
\tau_{\varphi}: C^{\infty}(M) \longrightarrow C^{\infty}\left(M^{A}, A\right), f \longmapsto-\left[\widetilde{\operatorname{ad(f)}]^{A}}(\varphi)\right.
$$

is a vector field on $M^{A}$. 
We denote

$$
\widetilde{\tau_{\varphi}}: C^{\infty}\left(M^{A}, A\right) \longrightarrow C^{\infty}\left(M^{A}, A\right)
$$

the unique $A$-linear derivation such that

$$
\widetilde{\tau_{\varphi}}\left(f^{A}\right)=\tau_{\varphi}(f)
$$

for any $f \in C^{\infty}(M)$. We have for $f \in C^{\infty}(M)$,

$$
\left.\widetilde{\tau_{f^{A}}}=\widetilde{[a d(f)}\right]^{A}
$$

and for $\varphi, \psi \in C^{\infty}\left(M^{A}, A\right)$ and for $a \in A$,

$$
\begin{aligned}
\widetilde{\tau}_{\varphi+\psi} & =\widetilde{\tau}_{\varphi}+\widetilde{\tau}_{\psi} ; \\
\widetilde{\tau}_{a \cdot \varphi} & =a \cdot \widetilde{\tau}_{\varphi} ; \\
\widetilde{\tau}_{\varphi \cdot \psi} & =\varphi \cdot \widetilde{\tau}_{\psi}+\psi \cdot \widetilde{\tau}_{\varphi} .
\end{aligned}
$$

For any $\varphi, \psi \in C^{\infty}\left(M^{A}, A\right)$, we let

$$
\{\varphi, \psi\}_{A}=\widetilde{\tau}_{\varphi}(\psi)
$$

In [3] we show that this bracket defines a structure of $A$-Poisson algebra on $C^{\infty}\left(M^{A}, A\right)$.

Theorem 3 If $M$ is a Poisson manifold with bracket $\{$,$\} , then \{,\}_{A}$ is the prolongation on $M^{A}$ of the structure of Poisson on $M$ defined by $\{$,$\} .$

\section{$3 \quad \widetilde{d}$-Poisson cohomology}

Proposition 4 When $M$ is a Poisson manifold with bracket $\{$,$\} , the map$

$$
C^{\infty}(M) \longrightarrow \operatorname{Der}_{A}\left[C^{\infty}\left(M^{A}, A\right)\right], f \longmapsto-[\widetilde{\operatorname{ad}(f)}]^{A}
$$

is a representation of $C^{\infty}(M)$ into $C^{\infty}\left(M^{A}, A\right)$.

We denote $\widetilde{d}$ the operator of cohomology associated to this representation. For any $p \in \mathbb{N}$,

$$
\Lambda_{\text {Pois }}^{p}\left(M^{A}, \sim\right)=\mathcal{C}^{p}\left[C^{\infty}(M), C^{\infty}\left(M^{A}, A\right)\right]
$$


denotes the $C^{\infty}\left(M^{A}, A\right)$-module of skew-symmetric multilinear forms of degree $p$ from $C^{\infty}(M)$ into $C^{\infty}\left(M^{A}, A\right)$. We have

$$
\Lambda_{\text {Pois }}^{0}\left(M^{A}, \sim\right)=C^{\infty}\left(M^{A}, A\right)
$$

We denote

$$
\Lambda_{\text {Pois }}\left(M^{A}, \sim\right)=\bigoplus_{p=0}^{n} \Lambda_{\text {Pois }}^{p}\left(M^{A}, \sim\right)
$$

Thus, for $\Omega \in \Lambda_{\text {Pois }}^{p}\left(M^{A}, \sim\right)$ and $f_{1}, \ldots, f_{p+1} \in C^{\infty}(M)$, we have

$$
\begin{aligned}
\tilde{d} \Omega\left(f_{1}, \ldots, f_{p+1}\right) & =\sum_{i=1}^{p+1}(-1)^{i}\left[\widetilde{a d\left(f_{i}\right)}\right]^{A}\left[\Omega\left(f_{1}, \ldots, \widehat{f}_{i}, \ldots, f_{p+1}\right)\right] \\
& +\sum_{1 \leq i<j \leq p+1}(-1)^{i+j} \Omega\left(\left\{f_{i}, f_{j}\right\}, f_{1}, \ldots, \widehat{f}_{i}, \ldots, \widehat{f}_{j}, \ldots, f_{p+1}\right)
\end{aligned}
$$

where $\widehat{f}_{i}$ means that the term $f_{i}$ is omitted.

When $\eta \in \Lambda_{\text {Pois }}^{p}(M)$, then

$$
\eta^{A}: C^{\infty}(M) \times \ldots \times C^{\infty}(M) \longrightarrow C^{\infty}\left(M^{A}, A\right),\left(f_{1}, \ldots, f_{p}\right) \longmapsto\left[\eta\left(f_{1}, \ldots, f_{p}\right)\right]^{A}
$$

is skew-symmetric multilinear forms of degree $p$ from $C^{\infty}(M)$ into $C^{\infty}\left(M^{A}, A\right)$ i.e

$$
\eta^{A} \in \Lambda_{\text {Pois }}^{p}\left(M^{A}, \sim\right)
$$

Thus

Proposition 5 For any $\eta \in \Lambda_{\text {Pois }}^{p}(M)$, we have $\widetilde{d} \eta^{A}=(d \eta)^{A}$. 
Proof. For any $f_{1}, \ldots, f_{p+1} \in C^{\infty}(M)$, we have

$$
\begin{aligned}
\left(\widetilde{d}^{A}\right)\left(f_{1}, \ldots, f_{p+1}\right) & =\sum_{i=1}^{p+1}(-1)^{i} \widetilde{\left.\operatorname{ad(f_{i})}\right]^{A}}\left(\eta^{A}\left(f_{1}, \ldots, \widehat{f}_{i}, \ldots, f_{p+1}\right)\right) \\
& +\sum_{1 \leq i<j \leq p+1}(-1)^{i+j} \eta^{A}\left(\left\{f_{i}, f_{j}\right\}, f_{1}, \ldots, \widehat{f}_{i}, \ldots, \widehat{f}_{j}, \ldots, f_{p+1}\right) \\
& =\sum_{i=1}^{p+1}(-1)^{i} \widetilde{\left.\operatorname{ad(f_{i})}\right]^{A}}\left(\eta\left(f_{1}, \ldots, \widehat{f}_{i}, \ldots, f_{p+1}\right)\right)^{A} \\
& +\sum_{1 \leq i<j \leq p+1}(-1)^{i+j}\left[\eta\left(\left\{f_{i}, f_{j}\right\}, f_{1}, \ldots, \widehat{f}_{i}, \ldots, \widehat{f}_{j}, \ldots, f_{p+1}\right)\right]^{A} \\
& =\sum_{i=1}^{p+1}(-1)^{i}\left\{f_{i}, \eta\left(f_{1}, \ldots, \widehat{f}_{i}, \ldots, f_{p+1}\right)\right\}^{A} \\
& +\sum_{1 \leq i<j \leq p+1}(-1)^{i+j}\left[\eta\left(\left\{f_{i}, f_{j}\right\}, f_{1}, \ldots, \widehat{f}_{i}, \ldots, \widehat{f}_{j}, \ldots, f_{p+1}\right)\right]^{A} \\
& =\left[(d \eta)\left(f_{1}, f_{2}, \ldots, f_{p+1}\right)\right]^{A} .
\end{aligned}
$$

That ends the proof.

Corollary 6 The 1-form $\eta^{A}$ is $\widetilde{d}$-closed i.e $\left(\widetilde{d}^{A}=0\right)$, if and only if $d \eta=0$. In particular when $\eta$ is a derivation of Poisson algebra $C^{\infty}(M)$.

Proof. Indeed, for $p=1$, we have

$$
\begin{aligned}
& \left.\left(\widetilde{d} \eta^{A}\right)(f, g)=\widetilde{[a d(f)}\right]^{A}\left[\eta^{A}(g)\right]-[\widetilde{a d(g)}]^{A}\left[\eta^{A}(f)\right]-\eta^{A}(\{f, g\}) \\
& =\{f, \eta(g)\}^{A}-\{g, \eta(f)\}^{A}-[\eta(\{f, g\})]^{A} \\
& =(\{f, \eta(g)\}-\{g, \eta(f)\}-\eta(\{f, g\}))^{A} \\
& =[d \eta(f, g)]^{A} \text {. }
\end{aligned}
$$

for any $f, g \in C^{\infty}(M)$.

Thus $\widetilde{d} \eta^{A}=0$ if qnd only if $d \eta=0$.

When $\eta$ is a derivation of Poisson algebra $C^{\infty}(M)$, we have $f, g \in C^{\infty}(M)$,

$$
\begin{aligned}
\eta(\{f, g\}) & =\{\eta(f), g\}+\{f, \eta(g)\} \\
& =\{f, \eta(g)\}-\{g, \eta(f)\}
\end{aligned}
$$

i.e

$$
\begin{aligned}
\left(\widetilde{d} \eta^{A}\right)(f, g) & =[d \eta(f, g)]^{A} \\
& =0 .
\end{aligned}
$$

That ends the proof. 
Proposition 7 If $\eta$ and $\eta^{\prime}$ both are cohomologous $d$-closed $p$-forms then $\eta^{A}$ and $\eta^{\prime A}$ both are cohomologous $\widetilde{d}$-closed $p$-forms.

Proof. For any $f_{1}, \ldots, f_{p} \in C^{\infty}(M)$ we have

$$
\begin{aligned}
{\left[\eta^{A}-\eta^{A}\right]\left(f_{1}, \ldots, f_{p}\right) } & =\eta^{A}\left(f_{1}, \ldots, f_{p}\right)-\eta^{A}\left(f_{1}, \ldots, f_{p}\right) \\
& =\left[\eta\left(f_{1}, \ldots, f_{p}\right)\right]^{A}-\left[\eta^{\prime}\left(f_{1}, \ldots, f_{p}\right)\right]^{A} \\
& =\left[\eta\left(f_{1}, \ldots, f_{p}\right)-\eta^{\prime}\left(f_{1}, \ldots, f_{p}\right)\right]^{A} \\
& =\left[\left(\eta-\eta^{\prime}\right)\left(f_{1}, \ldots, f_{p}\right)\right]^{A}
\end{aligned}
$$

If there exists $\nu \in \Lambda_{\text {Pois }}^{p-1}(M)$ such that

$$
\eta-\eta^{\prime}=d \nu
$$

then

$$
\begin{aligned}
{\left[\eta^{A}-\eta^{A}\right]\left(f_{1}, \ldots, f_{p}\right) } & =\left[\left(\eta-\eta^{\prime}\right)\left(f_{1}, \ldots, f_{p}\right)\right]^{A} \\
& =\left[d \nu\left(f_{1}, \ldots, f_{p}\right)\right]^{A} \\
& =\widetilde{d} \nu^{A}\left(f_{1}, \ldots, f_{p}\right)
\end{aligned}
$$

i.e

$$
\eta^{A}-\eta^{A}=\widetilde{d} \nu^{A}
$$

The cohomology class of the $d$-closed $p$-form $\eta$ induces the cohomology class of the $\widetilde{d}$-closed $p$-form $\eta^{A}$.

Let $Z_{P o i s}^{p}\left(M^{A}, \sim\right)$ be the set of $\widetilde{d}$-closed $p$-forms from $C^{\infty}(M)$ into $C^{\infty}\left(M^{A}, A\right)$ and $B_{\text {Pois }}^{p}\left(M^{A}, \sim\right)$ be the set of $\widetilde{d}$-exact $p$-forms from $C^{\infty}(M)$ into $C^{\infty}\left(M^{A}, A\right)$. We denote

$$
H_{\text {Pois }}^{p}\left(M^{A}, \sim\right)=Z_{\text {Pois }}^{p}\left(M^{A}, \sim\right) / B_{\text {Pois }}^{p}\left(M^{A}, \sim\right)
$$

For $p=0, \Lambda_{\text {Pois }}^{0}\left(M^{A}, \sim\right)=C^{\infty}\left(M^{A}, A\right)$. It is obvious that $H^{0}\left(M^{A}, \sim\right)$ is the center of $C^{\infty}\left(M^{A}, A\right)$ i.e the set

$$
\left.\left\{\phi \in\left(M^{A}, A\right) ; \widetilde{[a d(f)}\right]^{A}(\phi)=0 \text { for every } f \in C^{\infty}(M)\right\}
$$

For $p=1$, we have

$$
H_{\text {Pois }}^{1}\left(M^{A}, \sim\right)=0 .
$$




\section{$4 \widetilde{d_{A}}$-Poisson cohomology}

The map

$$
C^{\infty}\left(M^{A}, A\right) \longrightarrow \operatorname{Der}_{A}\left[C^{\infty}\left(M^{A}, A\right)\right], \varphi \longmapsto \widetilde{\tau_{\varphi}}
$$

is a representation of $C^{\infty}\left(M^{A}, A\right)$ into $C^{\infty}\left(M^{A}, A\right)$. We denote $\widetilde{d_{A}}$ the cohomology operator associated to this representation.

For any $p \in \mathbb{N}, \Lambda_{\text {Pois }}^{p}\left(M^{A}, \sim_{A}\right)=\mathcal{C}^{p}\left[C^{\infty}\left(M^{A}, A\right), C^{\infty}\left(M^{A}, A\right)\right]$ denotes the $C^{\infty}\left(M^{A}, A\right)$-module of skew-symmetric multilinear forms of degree $p$ on $C^{\infty}\left(M^{A}, A\right)$ into $C^{\infty}\left(M^{A}, A\right)$. We have

$$
\Lambda_{\text {Pois }}^{0}\left(M^{A}, \sim_{A}\right)=C^{\infty}\left(M^{A}, A\right) .
$$

We denote

$$
\Lambda_{\text {Pois }}\left(M^{A}, \sim_{A}\right)=\bigoplus_{p=0}^{n} \Lambda_{\text {Pois }}^{p}\left(M^{A}, \sim_{A}\right) .
$$

For $\Omega \in \Lambda_{\text {Pois }}^{p}\left(M^{A}, \sim_{A}\right)$ and $\varphi_{1}, \varphi_{2}, \ldots, \varphi_{p+1} \in C^{\infty}\left(M^{A}, A\right)$, we have

$$
\begin{aligned}
\widetilde{d_{A}} \Omega\left(\varphi_{1}, \ldots, \varphi_{p+1}\right) & =\sum_{i=1}^{p+1}(-1)^{i-1} \widetilde{\tau_{\varphi_{i}}}\left[\Omega\left(\varphi_{1}, \ldots, \widehat{\varphi}_{i}, \ldots, \varphi_{p+1}\right]\right. \\
& +\sum_{1 \leq i<j \leq p+1}(-1)^{i+j} \Omega\left(\left\{\varphi_{i}, \varphi_{j}\right\}_{A}, \varphi_{1}, \ldots, \widehat{\varphi}_{i}, \ldots, \widehat{\varphi_{j}}, \ldots, \varphi_{p+1}\right)
\end{aligned}
$$

i.e

$$
\begin{aligned}
\widetilde{d_{A}} \Omega\left(\varphi_{1}, \varphi_{2}, \ldots, \varphi_{p+1}\right) & =\sum_{i=1}^{p+1}(-1)^{i-1}\left\{\varphi_{i}, \Omega\left(\varphi_{1}, \ldots, \widehat{\varphi}_{i}, \ldots, \varphi_{p+1}\right\}_{A}\right. \\
& +\sum_{1 \leq i<j \leq p+1}(-1)^{i+j} \Omega\left(\left\{\varphi_{i}, \varphi_{j}\right\}_{A}, \varphi_{1}, \ldots, \widehat{\varphi}_{i}, \ldots, \widehat{\varphi_{j}}, \ldots, \varphi_{p+1}\right) .
\end{aligned}
$$

For $p=1$, we have

$$
\widetilde{d_{A}} \Omega(\varphi, \psi)=\{\Omega(\varphi), \psi\}_{A}+\{\varphi, \Omega(\psi)\}_{A}-\Omega\left(\{\varphi, \psi\}_{A}\right)
$$

for any $\varphi, \psi \in C^{\infty}\left(M^{A}, A\right)$. Thus

Corollary 8 The 1 -form $\Omega$ is $\widetilde{d_{A}}$-closed i.e $\widetilde{d_{A}} \Omega=0$ if, and only if,

$$
\Omega\left(\{\varphi, \psi\}_{A}=\{\Omega(\varphi), \psi\}_{A}+\{\varphi, \Omega(\psi)\}_{A}\right.
$$

i.e $\Omega$ is a derivation of the algebra $C^{\infty}\left(M^{A}, A\right)$. 
Let $Z_{P \text { ois }}^{p}\left(M^{A}, \sim_{A}\right)$ be the set of $\widetilde{d_{A}}$-closed $p$-forms from $C^{\infty}\left(M^{A}, A\right)$ into

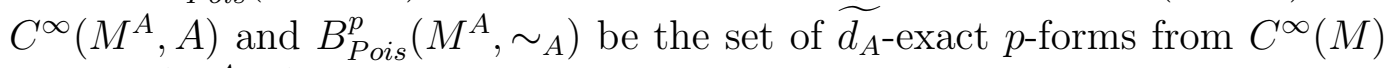
into $C^{\infty}\left(M^{A}, A\right)$. We denote

$$
H_{\text {Pois }}^{p}\left(M^{A}, \sim_{A}\right)=Z_{\text {Pois }}^{p}\left(M^{A}, \sim_{A}\right) / B_{\text {Pois }}^{p}\left(M^{A}, \sim_{A}\right) .
$$

For $p=0, \Lambda_{\text {Pois }}^{0}\left(M^{A}, \sim_{A}\right)=C^{\infty}\left(M^{A}, A\right)$. It is obvious that $H^{0}\left(M^{A}, \sim_{A}\right)$ is the center of $C^{\infty}\left(M^{A}, A\right)$ i.e the set

$$
\left\{\varphi \in C^{\infty}\left(M^{A}, A\right) ;\{\varphi, \phi\}_{A}=0 \text { for every } \phi \in C^{\infty}\left(M^{A}, A\right)\right\}
$$

For $p=1$, we have

$$
H_{\text {Pois }}^{1}\left(M^{A}, \sim_{A}\right)=0
$$

Acknowledgements: The first author thanks Deutscher Akademischer Austauschdientst (DAAD) for their financial support.

\section{References}

[1] B. G. R. Bossoto, E. Okassa, Champs de vecteurs et formes différentielles sur une variété de points proches, Archivum Mathematicum (Brno), 44(2008) 159-171.

[2] B. G. R. Bossoto, Structures de Jacobi sur une variété des points proches, Math. Vesnik. 62, 2 (2010), 155-167.

[3] B. G. R. Bossoto, E. Okassa, A-poisson structures on Weil bundles, Int., J. Contemp. Math. Sciences, Vol. 7, 2012, n¹6, 785-803.

[4] I. Kolar, P.W. Michor, and J. Slovak, Natural operations in differential geometry. Springer, 1993, 434 p.

[5] A. Lichnerowicz, Les variétés de Poisson et leurs algébres de Lie associées, J. Diff. Geom., 12 (1977), 253-300.

[6] A. Morimoto, Prolongation of connections to bundles of infinitely near points, J. Diff. Geom, t.11(1976), 479-498.

[7] E. Okassa, Prolongement des champs de vecteurs à des variétés de points proches, Annales Faculté des sciences de Toulouse, Vol. VIII, n³, 19861987, 349-366. 
[8] I. Vaisman, Lectures on the Geometry of Poisson Manifolds, Progress in Math.118, Birkhäuser Verlag, Basel, 1994.

[9] V. V. Shurygin, Smooth manifolds over local algebras and Weil bundles, J. Math. Sci., 108 (2) (2002), 249-294.

[10] A. Weil, Théorie des points proches sur les variétés différentiables, Colloq. Géom. Diff. Strasbourg (1953), 111-117.

Received: August 15, 2013 